\title{
On the Scalability of Snapshot Isolation ${ }^{\star}$
}

\author{
Masoud Saeida Ardekani ${ }^{1}$, Pierre Sutra ${ }^{2}$, Marc Shapiro ${ }^{3}$, and Nuno Preguiça ${ }^{4}$ \\ 1 Université Pierre et Marie Curie, Paris, France \\ ${ }_{2}$ University of Neuchâtel, Switzerland \\ 3 INRIA \& Université Pierre et Marie Curie, Paris, France \\ 4 CITI, Universidade Nova de Lisboa, Lisbon, Portugal
}

\begin{abstract}
Many distributed applications require transactions. However, transactional protocols that require strong synchronization are costly in large scale environments. Two properties help with scalability of a transactional system: genuine partial replication (GPR), which leverages the intrinsic parallelism of a workload, and snapshot isolation (SI), which decreases the need for synchronization. We show that under standard assumptions (data store accesses are not known in advance, and transactions may access arbitrary objects in the data store), it is impossible to have both SI and GPR. Our impossibility result is based on a novel decomposition of SI which proves that, like serializability, SI is expressible on plain histories.
\end{abstract}

\section{Introduction}

Large scale transactional systems have conflicting requirements. On the one hand, strong transactional guarantees are fundamental to many applications. On the other, remote communication and synchronization are costly and should be avoided. ${ }^{5}$

To maintain strong consistency guarantees while alleviating the cost of synchronization, Snapshot Isolation (SI) is a popular approach in both distributed database replications [1-3], and software transactional memories [4, 5]. Under SI, a transaction accesses its own consistent snapshot of the data, which is unaffected by concurrent updates. A read-only transaction always commits unilaterally and without synchronization. An update transaction synchronizes on commit to ensure that no concurrent conflicting transaction has committed before it.

Our first contribution is to prove that SI is equivalent to the conjunction of the following properties: (i) no cascading aborts, (ii) strictly consistent snapshots, i.e., a transaction observes a snapshot that coincides with some point in (linear) time, (iii) two concurrent write-conflicting update transactions never

\footnotetext{
* This work is partially supported by FCT/MCT projects PEst-OE/EEI/UI0527/2011 and PTDC/EIA-EIA/108963/2008, and the European Commission's Seventh Framework Program (FP7) under grant agreement No. 318809 (LEADS).

${ }^{5}$ We address general-purpose transactions, i.e., we assume that a transaction may access any object in the system, and that its read- and write-sets are not known in advance.
} 
both commit, and (iv) snapshots observed by transactions are monotonically ordered. Previous definitions of SI $[6,7]$ extend histories with abstract snapshot points. Our decomposition shows that in fact, like serializability, SI can be defined on plain histories [8].

Modern data stores replicate data for both performance and availability. Full replication does not scale, as every process must perform all updates. Partial replication (PR) aims to address this problem, by replicating only a subset of the data at each process. Thus, if transactions would communicate only over the minimal number of replicas, synchronization and computation overhead would be reduced. However, in the general case, the overlap of transactions cannot be predicted; therefore, many PR protocols perform system-wide global consensus [2, 3] or communication [9]. This negates the potential advantages of PR; hence, we require genuine partial replication [10] (GPR), in which a transaction communicates only with processes that replicate some object accessed in the transaction. With GPR, independent transactions do not interfere with each other, and the intrinsic parallelism of a workload can be thus exploited.

Our second contribution is to show that SI and GPR are incompatible. More precisely, we prove that an asynchronous message-passing system supporting GPR, even if it is failure-free, cannot compute monotonically ordered snapshots, nor strictly consistent ones.

This paper proceeds as follows. We introduce our system model in Section 2. Section 3 presents our decomposition of SI. Section 4 shows that GPR and SI are mutually incompatible. We discuss implications of this result and related work in Section 5. Section 6 closes this paper. Due to space constraints, some proofs are deferred to our companion technical report [11].

\section{Model}

This section defines the elements in our model and formalizes SI and GPR .

\subsection{Objects \& transactions}

Let Objects be a set of objects, and $\mathcal{T}$ be a set of transaction identifiers. Given an object $x$ and an identifier $i, x_{i}$ denotes version $i$ of $x$. A transaction $T_{i \in \mathcal{T}}$ is a finite permutation of read and write operations followed by a terminating operation, commit $\left(c_{i}\right)$ or abort $\left(a_{i}\right)$. We use $w_{i}\left(x_{i}\right)$ to denote transaction $T_{i}$ writing version $i$ of object $x$, and $r_{i}\left(x_{j}\right)$ to mean that $T_{i}$ reads version $j$ of object $x$. In a transaction, every write is preceded by a read on the same object, and every object is read or written at most once. ${ }^{6}$ We note $w s\left(T_{i}\right)$ the write set of $T_{i}$, i.e., the set of objects written by transaction $T_{i}$. Similarly, $r s\left(T_{i}\right)$ denotes the read set of transaction $T_{i}$. The snapshot of $T_{i}$ is the set of versions read by $T_{i}$. Two transactions conflict when they access the same object and one of them modifies it; they write-conflict when they both write to the same object.

\footnotetext{
${ }^{6}$ These restrictions ease the exposition of our results but do not change their validity.
} 


\section{$2.2 \quad$ Histories}

A complete history $h$ is a partially ordered set of operations such that (1) for every operation $o_{i}$ appearing in $h$, transaction $T_{i}$ terminates in $h,(2)$ for every two operations $o_{i}$ and $o_{i}^{\prime}$ appearing in $h$, if $o_{i}$ precedes $o_{i}^{\prime}$ in $T_{i}$, then $o_{i}<_{h} o_{i}^{\prime}$, (3) for every read $r_{i}\left(x_{j}\right)$ in $h$, there exists a write operation $w_{j}\left(x_{j}\right)$ such that $w_{j}\left(x_{j}\right)<_{h} r_{i}\left(x_{j}\right)$, and (4) any two write operations over the same objects are ordered by $<_{h}$. A history is a prefix of a complete history. For some history $h$, order $<_{h}$ is the real-time order induced by $h$. Transaction $T_{i}$ is pending in history $h$ if $T_{i}$ does not commit, nor abort in $h$. We note $\ll_{h}$ the version order induced by $h$ between different versions of an object, i.e., for every object $x$, and any two transactions $T_{i}$ and $T_{j}, x_{i} \ll_{h} x_{j}=w_{i}\left(x_{i}\right)<_{h} w_{j}\left(x_{j}\right)$. Following Bernstein et al. [12], we depict a history as a graph. We illustrate this with history $h_{1}$ below in which transaction $T_{a}$ reads the initial versions of objects $x$ and $y$, while transaction $T_{1}$ (respectively $T_{2}$ ) updates $x$ (resp. y). ${ }^{7}$

$$
\begin{aligned}
h_{1}=r_{a}\left(x_{0}\right) \longrightarrow & r_{1}\left(x_{0}\right) \cdot w_{1}\left(x_{1}\right) \cdot c_{1} \\
& r_{a}\left(y_{0}\right) \cdot c_{a} \longrightarrow r_{2}\left(y_{0}\right) \cdot w_{2}\left(y_{2}\right) \cdot c_{2}
\end{aligned}
$$

When order $<_{h}$ is total, we shall write a history as a permutation of operations, e.g., $h_{2}=r_{1}\left(x_{0}\right) \cdot r_{2}\left(y_{0}\right) \cdot w_{2}\left(y_{2}\right) \cdot c_{1} \cdot c_{2}$.

\subsection{Snapshot Isolation}

Snapshot isolation (SI) was introduced by Berenson et al. [8], then later generalized under the name GSI by Elnikety et al. [7]. In this paper, we make no distinction between SI and GSI.

Let us consider a function $\mathcal{S}$ which takes as input a history $h$, and returns an extended history $h_{s}$ by adding a snapshot point to $h$ for each transaction in $h$. Given a transaction $T_{i}$, the snapshot point of $T_{i}$ in $h_{s}$, denoted $s_{i}$, precedes every operation of transaction $T_{i}$ in $h_{s}$. A history $h$ is in SI if, and only if, there exists a function $\mathcal{S}$ such that $h_{s}=\mathcal{S}(h)$ and $h_{s}$ satisfies the following rules:

$$
\begin{aligned}
& \text { D1 (Read Rule) } \\
& \forall r_{i}\left(x_{j \neq i}\right), w_{k \neq j}\left(x_{k}\right), c_{k} \in h_{s}: \\
& \quad c_{j} \in h_{s} \\
& \wedge c_{j}<{ }_{h_{s}} s_{i} \\
& \wedge\left(c_{k}<{ }_{h_{s}} c_{j} \vee s_{i}<_{h_{s}} c_{k}\right)
\end{aligned}
$$

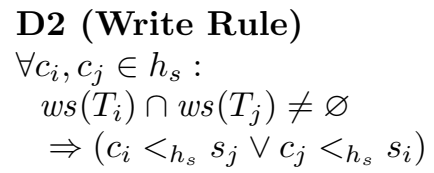

\subsection{System}

We consider a message-passing distributed system of $n$ processes $\Pi=\left\{p_{1}, \ldots, p_{n}\right\}$. We shall define our synchrony assumptions later. Following Fischer et al. [13], an execution is a sequence of steps made by one or more processes. During an execution, processes may fail by crashing. A process that does not crash is said

\footnotetext{
7 Throughout the paper, read-only transactions are specified with an alphabet subscript, and update transactions are shown with numeric subscript.
} 
correct; otherwise it is faulty. We note $\mathfrak{F}$ the refinement mapping [14] from executions to histories, i.e., if $\rho$ is an execution of the system, then $\mathfrak{F}(\rho)$ is the history produced by $\rho$. A history $h$ is acceptable if there exists an execution $\rho$ such that $h=\mathfrak{F}(\rho)$. We consider that given two sequences of steps $U$ and $V$, if $U$ precedes $V$ in some execution $\rho$, then the operations implemented by $U$ precedes (in the sense of $<_{h}$ ) the operations implemented by $V$ in the history $\mathfrak{F}(\rho) .^{8}$

\subsection{Partial Replication}

A data store $\mathcal{D}$ is a finite set of tuples $(x, v, i)$ where $x$ is an object (data item), $v$ a value, and $i \in \mathcal{T}$ a version. Each process in $\Pi$ holds a data store such that initially every object $x$ has version $x_{0}$. For an object $x$, Replicas $(x)$ denotes the set of processes, or replicas, that hold a copy of $x$. By extension for some set of objects $X, \operatorname{Replicas}(X)$ denotes the replicas of $X$; given a transaction $T_{i}$, $\operatorname{Replicas}\left(T_{i}\right)$ equals Replicas $\left(r s\left(T_{i}\right) \cup w s\left(T_{i}\right)\right)$.

We make no assumption about how objects are replicated. The coordinator of $T_{i}$, denoted $\operatorname{coord}\left(T_{i}\right)$, is in charge of executing $T_{i}$ on behalf of some client (not modeled). The coordinator does not know in advance the read set or the write set of $T_{i}$. To model this, we consider that every prefix of a transaction (followed by a terminating operation) is a transaction with the same id.

Genuine Partial Replication (GPR) aims to ensure that, when the workload is parallel, throughput scales linearly with the number of nodes [10]:

- GPR. For any transaction $T_{i}$, only processes that replicate objects accessed by $T_{i}$ make steps to execute $T_{i}$.

\subsection{Progress}

The read rule of SI does not define what is the snapshot to be read. According to Adya [6], "transaction $T_{i}$ 's snapshot point needs not be chosen after the most recent commit when $T_{i}$ started, but can be selected to be some (convenient) earlier point." To avoid that read-only transactions always observe outdated data, we add the following rule:

- Non-trivial SI. Consider an acceptable history $h$ and a transaction $T_{i}$ pending in $h$ such that the next operation invoked by $T_{i}$ is a read on some object $x$. Note $x_{j}$ the latest committed version of $x$ prior to the first operation of $T_{i}$ in $h$. Let $\rho$ be an execution satisfying $\mathfrak{F}(\rho)=h$. If $h . r_{i}\left(x_{j}\right)$ belongs to SI then there exists an execution $\rho^{\prime}$ extending $\rho$ such that in history $\mathfrak{F}\left(\rho^{\prime}\right)$, transaction $T_{i}$ reads at least (in the sense of $\ll_{h}$ ) version $x_{j}$ of $x$.

In addition, we consider that the system provides the following progress guarantees on transactions:

\footnotetext{
${ }^{8}$ Notice that since steps to implement operations may interleave, $<_{h}$ is not necessarily a total order.
} 
- Obstruction-free Updates. For every update transaction $T_{i}$, if $\operatorname{coord}\left(T_{i}\right)$ is correct then $T_{i}$ eventually terminates. Moreover, if $T_{i}$ does not writeconflict with some concurrent transaction, then $T_{i}$ eventually commits.

- Wait-free Queries. If $\operatorname{coord}\left(T_{i}\right)$ is correct and $T_{i}$ is a read-only transaction, then transaction $T_{i}$ eventually commits.

\section{Decomposing SI}

This section defines four properties, whose conjunction is necessary and sufficient to attain SI. We later use these properties in Section 4 to derive our impossibility result.

\subsection{Cascading Aborts}

Intuitively, a read-only transaction must abort if it observes the effects of an uncommitted transaction that later aborts. By guaranteeing that every version read by a transaction is committed, rules D1.1 and D1.2 of SI prevent such a situation to occur. In other words, these rules avoid cascading aborts. We formalize this property below:

Definition 1 (Avoiding Cascading aborts). History $h$ avoids cascading aborts, if for every read $r_{i}\left(x_{j}\right)$ in $h, c_{j}$ precedes $r_{i}\left(x_{j}\right)$ in $h . A C A$ denotes the set of histories that avoid cascading aborts.

\subsection{Consistent and Strictly Consistent Snapshots}

Consistent and strictly consistent snapshots are defined by refining causality into a dependency relation as follows:

Definition 2 (Dependency). Consider a history $h$ and two transactions $T_{i}$ and $T_{j}$. We note $T_{i} \triangleright T_{j}$ when $r_{i}\left(x_{j}\right)$ is in $h$. Transaction $T_{i}$ depends on transaction $T_{j}$ when $T_{i} \triangleright^{*} T_{j}$ holds. ${ }^{9}$ Transaction $T_{i}$ and $T_{j}$ are independent if neither $T_{i} \triangleright^{*} T_{j}$, nor $T_{j} \triangleright^{*} T_{i}$ hold.

This means that a transaction $T_{i}$ depends on a transaction $T_{j}$ if $T_{i}$ reads an object modified by $T_{j}$, or such a relation holds by transitive closure. To illustrate this definition, consider history $h_{3}=r_{1}\left(x_{0}\right) \cdot w_{1}\left(x_{1}\right) \cdot c_{1} \cdot r_{a}\left(x_{1}\right) \cdot c_{a} \cdot r_{b}\left(y_{0}\right) \cdot c_{b}$. In $h_{3}$, transaction $T_{a}$ depends on $T_{1}$. Notice that, even if $T_{1}$ causally precedes $T_{b}, T_{b}$ does not depend on $T_{1}$ in $h_{3}$.

We now define consistent snapshots with the above dependency relation. A transaction sees a consistent snapshot iff it observes the effects of all transactions it depends on [15]. For example, consider the history $h_{4}=r_{1}\left(x_{0}\right) \cdot w_{1}\left(x_{1}\right) \cdot c_{1} \cdot r_{2}\left(x_{1}\right)$ .$r_{2}\left(y_{0}\right) \cdot w_{2}\left(y_{2}\right) \cdot c_{2} \cdot r_{a}\left(y_{2}\right) \cdot r_{a}\left(x_{0}\right) \cdot c_{a}$ In this history, transaction $T_{a}$ does not see a

\footnotetext{
${ }^{9}$ We note $\mathcal{R}^{*}$ the transitive closure of some binary relation $\mathcal{R}$.
} 
consistent snapshot: $T_{a}$ depends on $T_{2}$, and $T_{2}$ also depends on $T_{1}$, but $T_{a}$ does not observe the effect of $T_{1}$ (i.e., $x_{1}$ ). Formally, consistent snapshots are defined as follows:

Definition 3 (Consistent snapshot). A transaction $T_{i}$ in a history $h$ observes a consistent snapshot iff, for every object $x$, if (i) $T_{i}$ reads version $x_{j}$, (ii) $T_{k}$ writes version $x_{k}$, and (iii) $T_{i}$ depends on $T_{k}$, then version $x_{k}$ is followed by version $x_{j}$ in the version order induced by $h\left(x_{k} \ll_{h} x_{j}\right)$. We write $h \in C O N S$ when all transactions in $h$ observe a consistent snapshot.

SI requires that a transaction observes the committed state of the data at some point in the past. This requirement is stronger than consistent snapshot. For some transaction $T_{i}$, it implies that (SCONSa) there exists a snapshot point for $T_{i}$, and (SCONSb) if transaction $T_{i}$ observes the effects of transaction $T_{j}$, it must also observe the effects of all transactions that precede $T_{j}$ in time. A history is called strictly consistent if both SCONSa and SCONSb hold.

To illustrate this, consider the following history: $h_{5}=r_{1}\left(x_{0}\right) \cdot w_{1}\left(x_{1}\right) \cdot c_{1} \cdot r_{a}\left(x_{1}\right)$ .$r_{2}\left(y_{0}\right) \cdot w_{2}\left(y_{2}\right) \cdot c_{2} \cdot r_{a}\left(y_{2}\right) \cdot c_{a}$. Because $r_{a}\left(x_{1}\right)$ precedes $c_{2}$ in $h_{5}, y_{2}$ cannot be observed when $T_{a}$ takes its snapshot. As a consequence, the snapshot of transaction $T_{a}$ is not strictly consistent. This issue is disallowed by SCONSa. Now, consider history $h_{6}=r_{1}\left(x_{0}\right) \cdot w_{1}\left(x_{1}\right) \cdot c_{1} \cdot r_{2}\left(y_{0}\right) \cdot w_{2}\left(y_{2}\right) \cdot c_{2} \cdot r_{a}\left(x_{0}\right) \cdot r_{a}\left(y_{2}\right) \cdot c_{a}$. Since $c_{1}$ precedes $c_{2}$ in $h_{6}$ and transaction $T_{a}$ observes the effect of $T_{2}$ (i.e., $y_{2}$ ), it should also observe the effect of $T_{1}$ (i.e., $x_{1}$ ). SCONSb prevents history $h_{6}$ to occur.

Definition 4 (Strictly consistent snapshot). Snapshots in history $h$ are strictly consistent, when for any committed transactions $T_{i}, T_{j}, T_{k \neq j}$ and $T_{l}$, the following two properties hold:

$$
\begin{array}{r}
\text { - } \forall r_{i}\left(x_{j}\right), r_{i}\left(y_{l}\right) \in h: r_{i}\left(x_{j}\right) \nless_{h} c_{l} \\
\text { - } \forall r_{i}\left(x_{j}\right), r_{i}\left(y_{l}\right), w_{k}\left(x_{k}\right) \in h: \\
c_{k}<{ }_{h} c_{l} \Rightarrow c_{k}<_{h} c_{j}
\end{array}
$$

We note SCONS the set of strictly consistent histories.

\subsection{Snapshot Monotonicity}

In addition, SI requires what we call monotonic snapshots. For instance, although history $h_{7}$ below satisfies SCONS, this history does not belong to SI. Indeed, since $T_{a}$ reads $\left\{x_{0}, y_{2}\right\}$, and $T_{b}$ reads $\left\{x_{1}, y_{0}\right\}$, there is no extended history that would guarantee the read rule of SI.

$$
\begin{aligned}
& h_{7}=r_{a}\left(x_{0}\right) \Longrightarrow r_{1}\left(x_{0}\right) \cdot w_{1}\left(x_{1}\right) \cdot c_{1} \longrightarrow r_{b}\left(x_{1}\right) \cdot c_{b} \\
& r_{b}\left(y_{0}\right) \Longrightarrow r_{2}\left(y_{0}\right) \cdot w_{2}\left(y_{2}\right) \cdot c_{2} \longrightarrow r_{a}\left(y_{2}\right) \cdot c_{a}
\end{aligned}
$$

SI requires monotonic snapshots. However, the underlying reason is intricate enough that some previous works [4, for instance] do not ensure this property, while claiming to be SI. Below, we introduce an ordering relation between snapshots to formalize snapshot monotonicity. 
Definition 5 (Snapshot precedence). Consider a history $h$ and two distinct transactions $T_{i}$ and $T_{j}$. The snapshot read by $T_{i}$ precedes the snapshot read by $T_{j}$ in history $h$, written $T_{i} \rightarrow T_{j}$, when $r_{i}\left(x_{k}\right)$ and $r_{j}\left(y_{l}\right)$ belong to $h$ and either (i) $r_{i}\left(x_{k}\right)<_{h} c_{l}$ holds, or (ii) transaction $T_{l}$ writes $x$ and $c_{k}<_{h} c_{l}$ holds.

For more illustration, consider histories $h_{8}=r_{1}\left(x_{0}\right) \cdot w_{1}\left(x_{1}\right) \cdot c_{1} \cdot r_{2}\left(y_{0}\right) \cdot w_{2}\left(y_{2}\right) \cdot r_{a}\left(x_{1}\right)$ .$c_{2} \cdot r_{b}\left(y_{2}\right) \cdot c_{a} \cdot c_{b}$ and $h_{9}=r_{1}\left(x_{0}\right) \cdot w_{1}\left(x_{1}\right) \cdot c_{1} \cdot r_{a}\left(x_{1}\right) \cdot c_{a} \cdot r_{2}\left(x_{1}\right) \cdot r_{2}\left(y_{0}\right) \cdot w_{2}\left(x_{2}\right) \cdot w_{2}\left(y_{2}\right) \cdot c_{2}$ $._{b}\left(y_{2}\right) . c_{b}$. In history $h_{8}, T_{a} \rightarrow T_{b}$ holds because $r_{a}\left(x_{1}\right)$ precedes $c_{2}$ and $T_{b}$ reads $y_{2}$. In $h_{9}, c_{1}$ precedes $c_{2}$ and both $T_{1}$ and $T_{2}$ modify object $x$. Thus, $T_{a} \rightarrow T_{b}$ also holds. We define snapshot monotonicity using snapshot precedence as follows:

Definition 6 (Snapshot monotonicity). Given some history $h$, if the relation $\rightarrow^{*}$ induced by $h$ is a partial order, the snapshots in $h$ are monotonic. We note MON the set of histories that satisfy this property.

According to this definition, both $T_{a} \rightarrow T_{b}$ and $T_{b} \rightarrow T_{a}$ hold in history $h_{7}$. Thus, history $h_{7}$ does not belong to MON.

Non-monotonic snapshots are observed under update serializability [16], that is when queries observe consistent state, but only updates are serializable.

\subsection{Write-Conflict Freedom}

Rule D2 of SI forbids two concurrent write-conflicting transactions from both committing. Since in our model we assume that every write is preceeded by a corresponding read on the same object, every update transaction depends on a previous update transaction (or on the initial transaction $T_{0}$ ). Therefore, under SI, concurrent conflicting transactions must be independent:

Definition 7 (Write-Conflict Freedom). A history $h$ is write-conflict free if two independent transactions never write to the same object. We denote by WCF the histories that satisfy this property.

\subsection{The decomposition}

Theorem 1 below establishes that a history $h$ is in SI iff (1) every transaction in $h$ sees a committed state, (2) every transaction in $h$ observes a strictly consistent snapshot, (3) snapshots are monotonic, and (4) $h$ is write-conflict free. A detailed proof appears in our companion technical report [11].

\section{Theorem 1. $S I=A C A \cap S C O N S \cap M O N \cap W C F$}

To the best of our knowledge, this result is the first to prove that SI can be split into simpler properties. Theorem 1 also establishes that SI is definable on plain histories. This has two interesting consequences: (i) a transactional system does not have to explicitly implement snapshots to support SI, and (ii) one can compare SI to other consistency criterion without relying on a phenomena based characterization. ${ }^{10}$

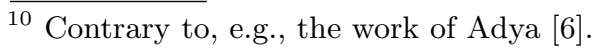




\section{The impossibility of SI with GPR}

This section leverages our previous decomposition result to show that SI is inherently non-scalable. In more details, we prove that none of MON, SCONSa or $\mathrm{SCONSb}$ is attainable in some asynchronous failure-free GPR system $\Pi$ when updates are obstruction-free and queries are wait-free. To prove these results, we first characterize below histories acceptable by $\Pi$.

Lemma 1. Let $h=\mathfrak{F}(\rho)$ be an acceptable history by $\Pi$ such that a transaction $T_{i}$ is pending in $h$. Note $X$ the set of objects accessed by $T_{i}$ in $h$. Only processes in Replicas $(X)$ make steps to execute $T_{i}$ in $\rho$.

Proof. (By contradiction.) Consider that a process $p \notin \operatorname{Replicas}(X)$ makes steps to execute $T_{i}$ in $\rho$. Since the prefix of a transaction is a transaction with the same id, we can consider an extension $\rho^{\prime}$ of $\rho$ such that $T_{i}$ does not execute any additional operation in $\rho^{\prime}$ and $\operatorname{coord}\left(T_{i}\right)$ is correct in $\rho^{\prime}$. The progress requirements satisfied by $\Pi$ imply that $T_{i}$ terminates in $\rho^{\prime}$. However, process $p \notin \operatorname{Replicas}(X)$ makes steps to execute $T_{i}$ in $\rho^{\prime}$. A contradiction to the fact that $\Pi$ is GPR.

We now state that monotonic snapshots are not constructable in $\Pi$. Our proof holds because objects accessed by a transaction are not known in advance.

Theorem 2. No asynchronous failure-free GPR system implements MON

Proof. (By contradiction.) Let us consider (i) four objects $x, y, z$ and $u$ such that for any two objects in $\{x, y, z, u\}$, their replica sets do not intersect; (ii) four queries $T_{a}, T_{b}, T_{c}$ and $T_{d}$ accessing respectively $\{x, y\},\{y, z\},\{z, u\}$ and $\{u, x\}$; and (iii) four updates $T_{1}, T_{2}, T_{3}$ and $T_{4}$ modifying respectively $x, y, z$ and $u$.

Obviously, history $r_{b}\left(y_{0}\right)$ is acceptable, and since updates are obstructionfree, $r_{b}\left(y_{0}\right) \cdot r_{2}\left(y_{0}\right) \cdot w_{2}\left(y_{2}\right) \cdot c_{2}$ is also acceptable. Applying that $\Pi$ satisfies nontrivial SI, we obtain that history $r_{b}\left(y_{0}\right) \cdot r_{2}\left(y_{0}\right) \cdot w_{2}\left(y_{2}\right) \cdot c_{2} \cdot r_{a}\left(x_{0}\right) \cdot r_{a}\left(y_{2}\right)$ is acceptable. Since $T_{a}$ must be wait-free, $h=r_{b}\left(y_{0}\right) \cdot r_{2}\left(y_{0}\right) \cdot w_{2}\left(y_{2}\right) \cdot c_{2} \cdot r_{a}\left(x_{0}\right) \cdot r_{a}\left(y_{2}\right) \cdot c_{a}$ is acceptable as well. Using a similar reasoning, history $h^{\prime}=r_{d}\left(u_{0}\right) \cdot r_{4}\left(u_{0}\right) \cdot w_{4}\left(u_{4}\right) \cdot c_{4}$ $r_{c}\left(z_{0}\right) . r_{c}\left(u_{4}\right) \cdot c_{c}$ is also acceptable. We note $\rho$ and $\rho^{\prime}$ respectively two sequences of steps such that $\mathfrak{F}(\rho)=h$ and $\mathfrak{F}\left(\rho^{\prime}\right)=h^{\prime}$.

System $\Pi$ is GPR. As a consequence, Lemma 1 tells us that only processes in Replicas $(x, y)$ make steps in $\rho$. Similarly, only processes in Replicas $(u, z)$ make steps in $\rho^{\prime}$. By hypothesis, Replicas $(x, y)$ and Replicas $(u, z)$ are disjoint. Applying a classical indistinguishably argument [13, Lemma 1], both $\rho^{\prime} . \rho$ and $\rho . \rho^{\prime}$ are admissible by $\Pi$. Thus, histories $h^{\prime} . h=\mathfrak{F}\left(\rho^{\prime} . \rho\right)$ and $h . h^{\prime}=\mathfrak{F}\left(\rho . \rho^{\prime}\right)$ are acceptable.

Since updates are obstruction-free, history $h^{\prime} \cdot h \cdot r_{3}\left(z_{0}\right) \cdot w_{3}\left(z_{3}\right) \cdot c_{3}$ is acceptable. Note $U$ the sequence of steps following $\rho^{\prime}$. $\rho$ with $\mathfrak{F}(U)=r_{3}\left(z_{0}\right) \cdot w_{3}\left(z_{3}\right) \cdot c_{3}$. Observe that by Lemma $1 \rho^{\prime} . \rho . U$ is indistinguishable from $\rho^{\prime} . U . \rho$. Then consider history $\mathfrak{F}\left(\rho^{\prime} . U . \rho\right)$. In this history, $T_{b}$ is pending and the latest version of object $z$ is $z_{3}$, As a consequence, because $\Pi$ satisfies non-trivial SI, there exists an extension of $\rho^{\prime} . U . \rho$ in which transaction $T_{b}$ reads $z_{3}$. From the fact that queries are 
wait-free and since $\rho^{\prime} . \rho . U$ is indistinguishable from $\rho^{\prime} . U . \rho$, we obtain that history $h_{1}=h^{\prime} . h . r_{3}\left(z_{0}\right) \cdot w_{3}\left(z_{3}\right) \cdot c_{3} \cdot r_{b}\left(z_{3}\right) \cdot c_{b}$ is acceptable. We note $U_{1}$ the sequence of steps following $\rho^{\prime} . \rho$ such that $\mathfrak{F}\left(U_{1}\right)$ equals $r_{3}\left(z_{0}\right) \cdot w_{3}\left(z_{3}\right) \cdot c_{3} \cdot r_{b}\left(z_{3}\right) \cdot c_{b}$.

With a similar reasoning, history $h_{2}=h^{\prime} \cdot h \cdot r_{1}\left(x_{0}\right) \cdot w_{1}\left(x_{1}\right) \cdot c_{1} \cdot r_{d}\left(x_{1}\right) \cdot c_{d}$ is acceptable. Note $U_{2}$ the sequence satisfying $\mathfrak{F}\left(U_{2}\right)=r_{1}\left(x_{0}\right) \cdot w_{1}\left(x_{1}\right) \cdot c_{1} \cdot r_{d}\left(x_{1}\right) \cdot c_{d}$.

Executions $\rho^{\prime} . \rho . U_{1}$ and $\rho^{\prime} . \rho . U_{2}$ are both admissible. Because $\Pi$ is GPR, only processes in Replicas $(y, z)$ (resp. Replicas $(x, u)$ ) make steps in $U_{1}$ (resp. $U_{2}$ ). By hypothesis, these two replica sets are disjoint. Applying again an indistinguishably argument, $\rho^{\prime} . \rho . U_{1} \cdot U_{2}$ is an execution of $\Pi$. Therefore, the history $\hat{h}=$ $\mathfrak{F}\left(\rho^{\prime} . \rho . U_{1} \cdot U_{2}\right)$ is acceptable. In this history, relation $T_{a} \rightarrow T_{b} \rightarrow T_{c} \rightarrow T_{d} \rightarrow T_{a}$ holds. Thus, $\hat{h}$ does not belong to MON. Contradiction.

Our next theorem states that SCONSb is not attainable. Similarly to Attiya et al. [17], our proof builds an infinite execution in which a query $T_{a}$ on two objects never terminates. We first define a finite execution during which we interleave between any two consecutive steps to execute $T_{a}$, a transaction updating one of the objects read by $T_{a}$. We show that during such an execution, transaction $T_{a}$ does not terminate successfully. Then, we prove that asynchrony allows us to continuously extend such an execution, contradicting the fact that queries are wait-free.

Definition 8 (Flippable execution). Consider two distinct objects $x$ and $y$, a query $T_{a}$ over both objects, and a set of updates $T_{j \in \llbracket 1, m \rrbracket}$ accessing $x$ if $j$ is odd, and $y$ otherwise. An execution $\rho=U_{1} V_{2} U_{2} \ldots V_{m} U_{m}$ where,

- transaction $T_{a}$ reads in history $h=\mathfrak{F}(\rho)$ at least version $x_{1}$ of $x$,

- for any $j$ in $\llbracket 1, m \rrbracket, U_{j}$ is the execution of transaction $T_{j}$ by processes $Q_{j}$,

- for any $j$ in $\llbracket 2, m \rrbracket, V_{j}$ are steps to execute $T_{a}$ by processes $P_{j}$, and

- both $\left(Q_{j} \cap P_{j}=\varnothing\right) \oplus\left(P_{j} \cap Q_{j+1}=\varnothing\right)$ and $Q_{j} \cap Q_{j+1}=\varnothing$ hold,

is called flippable.

Lemma 2. Let $\rho$ be an execution admissible by $\Pi$. If $\rho$ is flippable and histories accepted by $\Pi$ satisfy SCONSb, query $T_{a}$ does not terminate.

Proof. Let $h$ be the history $\mathfrak{F}(\rho)$. In history $h$ transaction $T_{j}$ precedes transaction $T_{j+1}$, it follows that $h$ is of the form $h=w_{1}\left(x_{1}\right) \cdot c_{1} * * \cdot w_{2}\left(y_{2}\right) \cdot c_{2} \cdot * \ldots$, where each symbol $*$ corresponds to either no operation, or to some read operation by $T_{a}$ on object $x$ or $y$.

Because $\rho$ is flippable, transaction $T_{a}$ reads at least version $x_{1}$ of object $x$ in $h$. For some odd natural $j \geq 1$, let $x_{j}$ denote the version of object $x$ read by $T_{a}$. Similarly, for some even natural $l$, let $y_{l}$ be the version of $y$ read by $T_{a}$. Assume that $j<l$ holds. Therefore, $h$ is of the form $h=\ldots w_{j}\left(x_{j}\right) \ldots w_{l}\left(y_{l}\right) \ldots$.

Note $k$ the value $l+1$, and consider the sequence of steps $V_{k}$ made by $P_{k}$ right after $U_{l}$ to execute $T_{a}$. Applying the definition of a flippable execution, we know that (F1) $\left(Q_{l} \cap P_{k}=\varnothing\right) \oplus\left(P_{k} \cap Q_{k}=\varnothing\right)$, and (F2) $Q_{l} \cap Q_{k}=\varnothing$. Consider now the following cases:

(Case $Q_{l} \cap P_{k}=\varnothing$.) It follows that $\rho$ is indistinguishable from the execution $\rho^{\prime \prime}=\ldots U_{j} \ldots V_{k} U_{l} U_{k} \ldots$ Then from fact $\mathrm{F} 2, \rho$ is indistinguishable from execution $\rho^{\prime}=\ldots U_{j} \ldots V_{k} U_{k} U_{l} \ldots$ 
(Case $P_{k} \cap Q_{k}=\varnothing$ ) With a similar reasoning, we obtain that $\rho$ is indistinguishable from $\rho^{\prime}=\ldots U_{j} \ldots U_{k} U_{l} V_{k} \ldots$

(Case $P_{k} \cap\left(Q_{l} \cup Q_{k}\right)=\varnothing$.) This case reduces to any of the two above cases. Note $h^{\prime}$ the history $\mathfrak{F}\left(\rho^{\prime}\right)$. Observe that since $\rho^{\prime}$ is indistinguishable from $\rho$, history $h^{\prime}$ is acceptable. In history $h^{\prime}, c_{k}<_{h^{\prime}} c_{l}$ holds. Moreover, $c_{j}<_{h^{\prime}} c_{k}$ holds by the assumption $j<l$ and the fact that $k$ equals $l+1$. Besides, operations $r_{i}\left(x_{j}\right), r_{i}\left(y_{l}\right)$ and $w_{k}\left(x_{k}\right)$ all belong to $h^{\prime}$. According to the definition of SCONSb, transaction $T_{a}$ does not commit in $h^{\prime}$. (The case $j>l$ follows a symmetrical reasoning to the case $l>j$ we considered previously.)

Theorem 3. No asynchronous failure-free GPR system implements SCONSb.

Proof. (By contradiction.) Consider two objects $x$ and $y$ such that Replicas $(x)$ and Replicas $(y)$ are disjoint. Assume a read-only transaction $T_{a}$ that reads successively $x$ then $y$. Below, we exhibit an execution admissible by $\Pi$ during which transaction $T_{a}$ never terminates. We build this execution as follows:

[Construction.] Consider some empty execution $\rho$. Repeat for all $i>=1$ : Let $T_{i}$ be an update of $x$, if $i$ is odd, and $y$ otherwise. Start the execution of transaction $T_{i}$. Since no concurrent transaction is write-conflicting with $T_{i}$ in $\rho$ and updates are obstruction-free, there must exist an extension $\rho . U_{i}$ of $\rho$ during which $T_{i}$ commits. Assign to $\rho$ the value of $\rho . U_{i}$. Execution $\rho$ is flippable. Hence, Lemma 2 tells us that transaction $T_{a}$ does not terminate in this execution. Consider the two following cases: (Case $i=1$ ) Because $\Pi$ satisfies non-trivial SI, there exists an extension $\rho^{\prime}$ of $\rho$ in which transaction $T_{a}$ reads at least version $x_{1}$ of object $x$. Notice that execution $\rho^{\prime}$ is of the form $U_{1} \cdot V_{2} . s \ldots$ where (i) all steps in $V_{2}$ are made by processes in Replicas $(x)$, and (ii) $s$ is the first step such that $\mathfrak{F}\left(U_{1} . V_{2} . s.\right)=r_{1}\left(x_{0}\right) \cdot w_{1}\left(x_{1}\right) \cdot c_{1} \cdot r_{a}\left(x_{1}\right)$. Assign $U_{1} \cdot V_{2}$ to $\rho$. (Case $\left.i>2\right)$ Consider any step $V_{i+1}$ to terminate $T_{a}$ and append it to $\rho$.

Execution $\rho$ is admissible by $\Pi$. Hence $\mathfrak{F}(\rho)$ is acceptable. However, in this history transaction $T_{a}$ does not terminate. This contradicts the fact that queries are wait-free.

SCONSa disallows some real time orderings between operations accessing different objects. Our last theorem shows that this property cannot be maintained under GPR.

Theorem 4. No asynchronous failure-free GPR system implements SCONSa.

Proof. (By contradiction.) Consider two distinct objects $x$ and $y$ such that Replicas $(x)$ and Replicas $(y)$ are disjoint. Let $T_{1}$ be an update accessing $y$, and $T_{a}$ be a query reading both objects.

Obviously, history $h=r_{a}\left(x_{0}\right)$ is acceptable. Note $U_{a}$ a sequence of steps satisfying $U=\mathfrak{F}\left(r_{a}\left(x_{0}\right)\right)$. Because $\Pi$ supports obstruction-free updates, we know the existence of an extension $U_{a} \cdot U_{1}$ of $U_{a}$ such that $\mathfrak{F}\left(U_{1}\right)=r_{1}\left(y_{0}\right) \cdot w_{1}\left(y_{1}\right) \cdot c_{1}$. By Lemma 1 , we observe that $U_{a} \cdot U_{1}$ is indistinguishable from $U_{1} \cdot U_{a}$. Then, since $\Pi$ satisfies non-trivial SI and read-only transactions are wait-free, there must exist an extension $U_{1} \cdot U_{a} \cdot V_{a}$ of $U_{1} \cdot U_{a}$ admissible by $\Pi$ and such that $\mathfrak{F}\left(V_{a}\right)=$ $r_{a}\left(y_{1}\right) \cdot c_{a}$. Finally, since $U_{a} \cdot U_{1}$ is indistinguishable from $U_{1} \cdot U_{a}$ and $U_{1} \cdot U_{a} \cdot V_{a}$ is admissible, $U_{a} \cdot U_{1} \cdot V_{a}$ is admissible too. The history $\mathfrak{F}\left(U_{a} \cdot U_{1} \cdot V_{a}\right)$ is not in SCONSa. Contradiction. 
As a consequence of the above, no asynchronous system, even if it is failurefree, can support both GPR and SI. In particular, even if the system is augmented with failure detectors [18], a common approach to model partial synchrony, SI cannot be implemented under GPR. This fact strongly hinders the usage of SI at large scale. In the following section, we further discuss implications of this result.

\section{Discussion}

A straightforward corollary of any of the theorems we proved in Section 4 is that neither strict serializability [19], nor opacity [20] is attainable under GPR. In the case of opacity, this answers negatively to a problem posed by Peluso et al. [21].

The classical (non-genuine) solution for building strictly consistent monotonic snapshots is to use total order broadcast (e.g., $[2,3]$ ).

When a transaction declares objects it accesses in advance, a GPR system can install a snapshot just after the start of the transaction. As a consequence, such an assumption sidesteps our impossibility result.

A transactional system $\Pi$ is permissive with respect to a consistency criterion $\mathcal{C}$ when every history $h \in \mathcal{C}$ is acceptable by $\Pi$. Permissiveness [22] measures the optimal amount of concurrency a system allows. If we consider again histories $h_{1}$ and $h_{2}$ in the proof of Theorem 2, we observe that both histories are serializable. Hence, every system permissive with respect to SER accepts both histories. By relying on the very same argument as the one we exhibit to close the proof of Theorem 2, we conclude that no transactional system is both GPR and permissive with respect to SER. For instance, none of the systems presented in [10, 23] accept history $h_{10}=r_{1}\left(x_{0}\right) \cdot w_{1}\left(x_{1}\right) \cdot c_{1} \cdot r_{2}\left(x_{0}\right) \cdot r_{2}\left(y_{0}\right) \cdot w_{2}\left(y_{2}\right) \cdot c_{2}$.

Recent distributed transactional systems (e.g., [9, 24]) support weaker consistency criteria than SI or SER. In particular, Walter [9] supports Parallel Snapshot Isolation (PSI). PSI is weaker than SI, and allows snapshots to be nonmonotonic. But, it still requires SCONSa to be ensured. Sovran et al. justify the use of PSI in Walter by the fact that SI is too expensive in a geographically distributed environment [9, page 4]. Our impossibility result establishes that, in order to scale, a transactional system needs supporting both non-monotonic and non-strictly consistent snapshots.

\section{Conclusion}

Partial replication and genuineness are two key factors of scalability in replicated systems. This paper shows that ensuring snapshot isolation (SI) in a genuine partial replication system is impossible. To state this impossibility result, we prove that SI is decomposable into a set of simpler properties. We show that two of these properties, namely snapshot monotonicity and strictly consistent snapshots cannot be ensured. As a corollary of our results, a GPR system cannot support neither strict serializability, nor opacity. 


\section{Bibliography}

[1] K. Daudjee et al., "Lazy database replication with snapshot isolation," in $V L D B$, Sep. 2006, pp. 715-726.

[2] D. Serrano et al., "Boosting Database Replication Scalability through Partial Replication and 1-Copy-Snapshot-Isolation," in PRDC, Dec. 2007, pp. 290-297.

[3] J. E. Armendáriz-Iñigo et al., "SIPRe: a partial database replication protocol with SI replicas," in SAC, Mar. 2008, pp. 2181-2185.

[4] A. Bieniusa et al., "Consistency in hindsight: A fully decentralized stm algorithm," in IPDPS, Apr. 2010, pp. 1-12.

[5] T. Riegel et al., "Snapshot isolation for software transactional memory," in TRANSACT, Jun. 2006.

[6] A. Adya, "Weak Consistency: A Generalized Theory and Optimistic Implementations for Distributed Transactions," Ph.D., MIT, Mar. 1999.

[7] S. Elnikety et al., "Database Replication Using Generalized Snapshot Isolation," in $S R D S$, Oct. 2005, pp. 73-84.

[8] H. Berenson et al., "A critique of ansi sql isolation levels," in SIGMOD, May 1995, pp. 1-10.

[9] Y. Sovran et al., "Transactional storage for geo-replicated systems," in SOSP, Oct. 2011, pp. 385-400.

[10] N. Schiper et al., "P-store: Genuine partial replication in wide area networks," in SRDS, Nov. 2010, pp. 214-224.

[11] M. Saeida Ardekani et al., "Non-Monotonic Snapshot Isolation," INRIA, Tech. Rep. RR-7805, Oct. 2012.

[12] P. Bernstein et al., Concurrency Control and Recovery in Database Systems. Addison Wesley Publishing Company, 1987.

[13] M. J. Fischer et al., "Impossibility of distributed consensus with one faulty process," Journal of the ACM, vol. 32, no. 2, pp. 374-382, Apr. 1985.

[14] M. Abadi et al., "The existence of refinement mappings," Theory Computer Science, vol. 82, pp. 253-284, May 1991.

[15] A. Chan et al., "Implementing Distributed Read-Only Transactions," IEEE Transactions on Software Engineering, vol. SE-11, no. 2, pp. 205-212, Feb. 1985.

[16] H. Garcia-Molina et al., "Read-only transactions in a distributed database," $A C M$ Trans. Database Syst., vol. 7, no. 2, pp. 209-234, Jun. 1982.

[17] H. Attiya et al., "Inherent limitations on disjoint-access parallel implementations of transactional memory," in SPAA, 2009, pp. 69-78.

[18] T. D. Chandra et al., "Unreliable failure detectors for reliable distributed systems," Journal of the ACM, vol. 43, no. 2, pp. 225-267, 1996.

[19] C. H. Papadimitriou, "The serializability of concurrent database updates," Journal of the ACM, vol. 26, no. 4, pp. 631-653, Oct. 1979.

[20] R. Guerraoui et al., "On the correctness of transactional memory," in PPoPP, 2008, pp. 175-184.

[21] S. Peluso et al., "Genuine replication, opacity and wait-free read transactions: can a stm get them all?" in WTTM, Madeira, Portugal, Jul. 2012.

[22] R. Guerraoui et al., "Permissiveness in transactional memories," in DISC, Sep. 2008, pp. 305-319.

[23] D. Sciascia et al., "Scalable deferred update replication," in DSN, Jun. 2012, pp. $1-12$.

[24] S. Peluso et al., "When scalability meets consistency: Genuine multiversion update-serializable partial data replication," in ICDCS, Jun. 2012, pp. 455-465. 